\title{
Les contraceptifs oraux sont-ils inoffensifs pour le foie?
}

La regulation des naissances est un problème social si brûlant que les intéressés ont salué avec enthousiasme l'apparition des contraceptifs oraux, simples à utiliser et efficaces. Les médecins étaient au debut un peu réticents devant la consummation presque quotidienne de produits stéroïdiens pendant des années; mais des contrôles pro-longés, avant le lancement de ces medicaments sur le marché, avaient fourni toute garantie.

Certains effets secondaires existent cependant, que ,/. Meylan a passes en revue récemment (Médecíne et Hygiene du 26 mai 1965, p. 539) en se fondant sur 18 enquêtes englobant plusieurs milliers de femmes observées pendant un total de 434000 cycles!

Des métrorragies surviennent parfois particulièrement au cours des trois premiers mois d'utilisation des nor-stéroïdes; par la suite, ces medicaments régularisent au contraire le cycle menstruel et diminuent son abondance.

Des nausées se rencontrent également au debut de l'emploi des contraceptifs; on signale encore, plus rarement, des céphalées, des vertiges, des crampes. Une augmentation de poids s'observe parfois, liée soit à une retention hydrosaline consecutive soit à Thyperaldo-stéronisme, soit à $\Gamma$ effet anabolisant de ces hormones.

Enfin on a publié un ou deux cas de phlébite, et les thrombocytes auraient en eífet une capacité d'adhérence («adhesiveness») supérieure sous Гinfluence des nor-stéroïdes.

A côté de ces inconvénients mineurs, en relation vraisemblable avec les contraceptifs oraux, un assez grand nombre de femmes consultent pour des troubles digestifs d'apparition récente, se demandant - et posant la question au spécialiste - si leurs malaises ne proviennent pas des norstéroïdes qu'elles utilisent depuis quelques mois ou années ?

En effet, les progestatifs de synthèse peuvent élever, pendant la durée du traitement - et celui-ci n'est pas destine à être bref! - le taux des transaminases hépatiques, des phosphatases alcalines et du test de McLagan au thymol, ainsi que la retention de brome-sulfone-phtaléine; et au cours de Гannée 1964, plusieurs auteurs avaient sou-

\section{Editorial}

61

ligné l'hépatotoxicité potentielle des stéroïdes alkylés en C.17-7. et rapporté quelques observations de soufFrances cliniques, et surtout humorales. Dans $\Gamma$ observation d'une jaunísse consecutive à $\Gamma$ ingestion de Lyndiol, en Suède, G. Cullberg et coll. (Brit. Med. J.; 13 mars 1965' p. 655) ont trouvé, à la ponction-biopsie, Гimage histologique d'une cholestase intrahépatique, avec alteration parenchymateuse.

Cependant, les æstrogènes sont prescrits depuis des dizaines d'années (à des doses bien supérieures à celles des contraceptifs!) et sans avoir entraîné d'inconvénient dans le domaine du foie. Faut-il alors incriminer la synergie des stéroïdes cestrogéniques et progesta-tifs?

Aussi le British Medical Journal a-t-il consacré tout récemment à cette question trois articles de son numéro du 29 mai 1965.

A. Eísalo, P. A., Järvínen et T. Luukkaínen d'Helsinki (p. 1416) avaient jeté le cri d'alarme en 1964. Depuis, ils ont suivi 109 femmes âgées de 17 à 52 ans et observent des elevations 
modérées des trans-aminases et de la BSP, respectivement chez $4 \%$ à $18 \%$ pour le premier test et pour la BSP, chez $19 \%$ à $49 \%$ des femmes. Mais tout rentre dans $\Gamma$ ordre en 4 semaines après Гarrêt du medicament. U. Larsson-Cohn, d'Uppsala (p. 1414), obtient des chiffres analogues. Enfin G. I. M. Swyer et V. Little (p. 1412) ont suivi de près 12 femmes préménopausiques pendant 3 à 6 ans de consummation régu-lière de progestatifs. Ils trouvent seulement chez $\Gamma$ une des patientes une hyperbilirubinémie modeste $(17 \mathrm{mg} / \mathrm{l})$, chez une autre une trans-aminase G. 0 . à peine élevée (30 U. Karmen), et tous les autres tests fonctionnels hépatiques dans les limites normales.

Le nombre insignifiant d'incidents signalés depuis $\Gamma$ emploi des contraceptifs oraux par des millions de femmes, et les résultats des recherches signalées plus haut, paraissent autoriser les médecins à rassurer leurs malades quant aux inconvénients de ces produits pour leur foie. M. D. 